\title{
Diabetic myelopathy
}

National Diabetes Information Clearinghouse (NDIC)

\section{Definitions}

Diabetes

Defined by National Diabetes Information Clearinghouse (NDIC)

\section{Source}

National Diabetes Information Clearinghouse (U.S.). (2009). The diabetes dictionary. [Bethesda, Md.]: U.S. Dept. of Health and Human Services, National Institutes of Health, National Institute of Diabetes and Digestive and Kidney Diseases, National Diabetes Information Clearinghouse.

Damage to the spinal cord found in some people with diabetes. 